\title{
Factors influencing growth of children aged 12-24 months in the Tanga region, Tanzania
}

Ingvild Skålnes Elverud ${ }^{1}$, Ketil Størdal ${ }^{2,3}$, Mercy Chiduo ${ }^{4}$, Claus Klingenberg ${ }^{1,5}$

${ }^{1}$ Paediatric Research Group, Faculty of Health Sciences, University of Troms $\varnothing$, Troms $\varnothing$,

Norway

${ }^{2}$ Paediatric Department, Ostfold Hospital Trust, Grålum, Norway

${ }^{3}$ Norwegian Institute of Public Health, Oslo, Norway

${ }^{4}$ National Institute for Medical Research, Tanga Research Centre, Tanga, Tanzania

${ }^{5}$ Department of Paediatrics, University Hospital of North-Norway, Troms $\emptyset$, Norway

\section{Corresponding author:}

Claus Klingenberg, $\mathrm{MD}, \mathrm{PhD}$

Dept. of Paediatrics, University Hospital of North-Norway, N-9038 Troms $\emptyset$, Norway

Tel: +47 77626000. Fax: +47 77626369

Email: claus.klingenberg@unn.no

Total words in abstract: 241

Total words in the main text of the paper: 2478

Number of tables: 4

Number of figures: 1

Number of references: 19 


\begin{abstract}
Background: The first 1,000 days of life, from conception to the second birthday, offer a unique window of opportunity for optimal growth, critical for future health. The primary aim of this study was to analyse growth of children between 12 to 24 months age in Tanzanian children, and to explore possible predictors for growth.
\end{abstract}

Methods: Observational, cross-sectional study performed between March-April 2017. Eligible children, and their mothers, attended routine follow-up at two health clinics in Tanga, Tanzania. At the study day, the child's weight and height were recorded. The mothers answered a structured interview regarding breastfeeding, immunization and socioeconomic conditions.

Results: We recruited 300 mother-child pairs. Median (interquartile range-IQR) at study visit was $16(14-20)$ months. Mothers reported that $170(57 \%)$ of their children were exclusively breastfed for a minimum of 6 months; median (IQR) 6 (4-6) months. Using the WHO standard growth curves, mean weight-for-age Z-score was -0.30 and mean length-for-age Zscore was -0.47 . Children whose mothers had higher education had higher Z-scores for weight and length compared to children of mothers with lower education. Education remained the most important predictor for growth also after adjusting for other variables. Overall, 48/300 (16\%) were moderate-severe stunted and 25/300 (8.4\%) had moderate-severe underweight. Conclusion: Children aged 12-24 months in this region of Tanzania had weight and height below the WHO-standard. Higher educated mothers had children with better growth parameters. Duration of exclusive breastfeeding was long, but did not predict growth parameters. 


\section{INTRODUCTION}

Optimal infant and child feeding practise, including breastfeeding, promotes growth and cognitive development, reduces the burden of infectious diseases, improves survival and increases adult income [1-4]. Globally it has been estimated that around 13\% of deaths among infants are due to suboptimal feeding practices $[2,5,6]$. The health benefit gained from breastfeeding is inversely proportional to the populations socioeconomic level [7]. There is an increasing awareness about the positive effects of breastfeeding, and globally the number of children being exclusively breastfed is increasing $[5,8]$. Still, between $0.8-1.4$ million lives could be saved each year if every child between the 0-23 months of age was optimally breastfed, and $10 \%$ of the disease burden in children under five years of age could be reduced $[3,5,7]$.

Tanzania is a stable country not affected by serious conflicts, war or famine [9]. Still, Tanzania is on the United Nations list of the least developed countries [10], and the World Health Organization (WHO) reports that many children under five years of age are underweight (13.7\%), stunted (34.5\%) or wasted (4.5\%) [11]. A study from rural Tanzania in 2010 revealed that child-feeding practices was not in line with WHO recommendations, and the prevalence of underweight children had been unchanged since 1987-1988 [12]. Recent studies from Tanzania found that malnutrition was associated with suboptimal cognitive, communicative and motor development $[4,13]$. Overall, in Sub-Saharan Africa there has been an increase in exclusive breastfeeding at 6 months from $22 \%$ in 1996 to $30 \%$ in 2006 [5, 8], but there is limited recent data from Tanzania.

The primary aim of the study was to describe growth of children aged 12 to 24 months North-East Tanzania. Specifically, we aimed to analyse possible associations between exclusive breastfeeding, other maternal factors and child growth. 


\section{METHODS}

\section{Design and setting}

This was an observational, cross-sectional study performed in Tanga, a coastal city of around 270000 inhabitants in North-East Tanzania. The major economy in Tanga is agriculture, small industry, business and fishing. In the current study, children between 12 and 24 months of age, and their mothers, attending two different health clinics for routine follow-up were eligible for inclusion. Exclusion criteria were children in other age groups and children attending with another caretaker than the mother. We did not ask about maternal HIV-status, and thus perinatal HIV-exposure was not an exclusion criterion. We aimed to include 300 mother-child pairs during the 5-week study period between March-April 2017. All mothers attending the health clinics on normal working days between 8 and 12 am were asked to participate.

\section{Data collection}

Mother-child pairs meeting inclusion criteria were asked for consent to participate in the study. We interviewed participating mothers using a standard questionnaire in Swahili, focusing on duration of exclusive breastfeeding, mixed feeding, ongoing breastfeeding at study visit, maternal age, education and occupation, number of pregnancies, parity and year of birth of each child (Appendix). Each interview lasted approximately 5-10 minutes. Local health care professionals helped as interpreters.

The child's weight from five previous monthly visits were collected from the "underfives clinic cards", height had not been routinely recorded. At the study day, children were weighed to the closest 100 grams using the mechanical hanging scale at the two health clinics (Waymaster, Reading, UK or Salter, Melbourne, Australia). In addition, height was measured to the closest $\mathrm{cm}$ by one of the authors (ISE) using a measuring tape. There was no length 
board available for measuring recumbent length, and all height measurements in these children aged 12-24 months were therefore added $0.7 \mathrm{~cm}$ to convert it to length.

\section{Data handling and statistical methods}

The first author manually entered all data from the interviews in a paper case-record file (Appendix). Subsequently all data were reviewed and checked for consistency, then registered and analyzed using IBM SPSS Statistics 25 (IBM, New York, USA). Weight-for-age Z-score (WAZ) and length-for-age Z-score (LAZ) were calculated using WHO child growth standards [14]. Underweight and stunting were defined as a WAZ or LAZ $<-2.0[14,15]$. We also calculated changes in WAZ over the last 5 months $(\Delta-\mathrm{WAZ})$ by subtracting the Z-score from study visit to the Z-score five months prior to study visit. $\Delta$-WAZ was split into three categories; declining weight $(\Delta-\mathrm{WAZ}<-0.3)$, stable weight $(\Delta-\mathrm{WAZ}-0.3$ to 0.3$)$ and increasing weight $(\Delta-\mathrm{WAZ}>0.3)$. Interval data were tested for normality using the Kolmogorov-Smirnov test. Normally distributed data are presented using means and standard deviations (SD), and non-normally distributed data are presented using medians and interquartile ranges (IQR). We used the T-test or Mann-Whitney U test for comparison of normal and non-normal distributed interval data, respectively. We used a linear regression model to analyze predictors that we, based on clinical and scientific experience, know may affect growth in childhood. We first performed univariate analysis. We then adjusted the results using a multiple linear regression analysis including only predictors with $\mathrm{p}<0.20$ in the univariate analysis. $\mathrm{P}$ values $<0.05$ were considered significant.

\section{Ethics}

Study approval was granted by the Medical Research Coordinating Committee, under the Tanzanian National Institute of Medical Research (NIMR/HQ/R.8a/Vol. IX/2349). All mothers consented to study participation by signing their name or printing their fingerprint on consent forms in Swahili and English. 


\section{RESULTS}

Three-hundred mother-child pairs were included in the study. None of the approached and eligible mothers declined study participation. Additional 27 mother-child pairs consented to participate but were later excluded as it turned out that the children were either below 12 months or above 24 months of age. Background data on all 300 mother-child pairs, including anthropometric measures at study visit, are shown in Table 1. Thirteen (4\%) mothers had given birth to five children or more. Three (1\%) mothers were below 20 years of age, and none were below 18 years of age. The children were not medically examined by the study authors, but the overall impression was that the majority were in a clinical stable condition without acute illness, and we did not observe children with obvious congenital anomalies. Figure 1 displays the proportion of children who had a declining (44\%), stable (42\%) or increasing (14\%) $\Delta$-WAZ over the last five months before data collection.

Around $3 / 4$ of the mothers reported exclusive breastfeeding $\geq 4$ months (Table 1 ). Multiparous mothers reported exclusive breastfeeding for median (IQR) 6 (4-6) months compared to median (IQR) 5 (3-6) months in primiparous mothers, $\mathrm{p}=0.001$. At study visit, $132(44 \%)$ of all 300 children were $\geq 18$ months of age, and 91/132 (69\%) of their mothers reported ongoing breastfeeding.

Educational level was dichotomized into "low maternal education" (no education or only primary school; $\mathrm{n}=187$ ) and "high maternal education" (secondary school or higher; $\mathrm{n}$ $=113$ ). There were no differences in duration of exclusive breastfeeding between the two groups (Table 2). WAZ and LAZ were significantly higher in children of mothers with high educational level compared with children of mothers with low educational level (Table 2). In the linear regression model, maternal education remained significantly associated with both WAZ and LAZ, after adjusting for other possible predictors for child growth (Table 3). 
Increasing maternal age, which was closely correlated to maternal education level (R $0.035, \mathrm{p}$ $=0.001)$, was also associated with better WAZ, but not LAZ. No other variable were associated with WAZ or LAZ at study visit. There was no significant difference in WAZ, LAZ or exclusive breastfeeding duration among children with mothers working at home ("housewife") and mothers working outside the home (data not shown).

In Table 4 we present prevalence of children that were stunted and underweight [14] in the current study and compare with data from the 2014 Tanzanian national nutrition survey [15]. Overall, data from Tanga in 2017 showed that the prevalence's of moderate-severe stunting and/or underweight were well below the national level in 2014.

\section{DISCUSSION}

This study reports data on breastfeeding practices and growth among children between 12 and 24 months of age in the Tanga region of Tanzania in 2017. The main finding was the significant association between growth and maternal education level; children whose mothers had secondary or higher education had significantly higher weight and length compared to children whose mothers had lower education. In general, rates of exclusive breastfeeding up to 6 months of age were high and duration of partial breastfeeding was long among mothers participating in this study. However, we found no distinct association between growth and duration of exclusive breastfeeding.

More than half of the mothers in this study reported that their child had been exclusively breastfed for six months. This is a much higher proportion than has recently been reported in Tanzania [15] and at a global level [3]. Almost $87 \%$ of the mothers in our study were still breastfeeding at 12 months, and these data are similar to national Tanzanian numbers [15]. A meta-analysis of studies from 127 low- and middle-income countries found it 
more common to breastfeed for at least 12 months in these countries, compared to in highincome countries [3]. Some data have also suggested that being a primiparous mother is a risk factor for only partial breastfeeding [2]. In line with this, we found that primiparous women were breastfeeding for shorter duration than multiparous women.

The mean Z-scores for weight and height were below the average according to the WHO child growth standards [14]. Moreover, around four in 10 children in our study had decreasing WAZ over the last five months before study visit, which means they were not gaining weight as they should [14]. This suggests that conditions for optimal growth, including adequate nutrition, may be challenging in the Tanga region. Indeed, in the national Tanzanian nutrition survey from 2014, the Tanga region was among the regions with the highest rates of acute malnutrition [15].

The prevalence of stunted and underweight children in this study was still markedly lower than in the similar age group covered in the national nutrition survey of 2014 [15], and in a study from central Tanzania in 2015 nearly half of the child population below five years was stunted [16]. This indicates considerable regional differences in Tanzania where current data from Tanga shows a better situation, and maybe also an improvement since 2014 [15]. The MAL-ED longitudinal birth cohort study [17], including children from a rural site in Tanzania, found that $16 \%$ of children were stunted at enrolment and the prevalence of stunting increased to $72 \%$ at 24 months [4], much higher numbers than we found in the current study. We speculate that the general long duration of exclusive breastfeeding and partial found in our study may be a reason for better growth than reported in some other regions of Tanzania.

In the MAL-ED study, children living in households with lower socioeconomic status had higher risk of being stunted [17]. This corresponds with findings from our study where the most important factor associated with growth at study visit was the maternal education 
level. Similar results were also identified in central Tanzania, where children of mothers who had completed seven years or more of education had a lower prevalence of stunting compared to children of mothers with seven years education or less [16]. Mothers with higher education may have greater knowledge about child health care and government recommendations. Moreover, women of higher education may not only have a higher income and as a result, they afford more nutritious foods, but they may also better utilize antenatal care, child health care and other health benefits. Higher maternal education has also been associated with improved protection against tuberculosis among HIV-exposed children in Tanzania [18].

In contrast, maternal education level did not predict the duration of exclusive breastfeeding, and indeed maternal education level was a significant predictor for growth even after adjustment for breastfeeding duration. Our data indicates that infant breastfeeding practice is similar in the education groups. The strong association between growth and maternal education persisted after adjustment for breastfeeding, this makes it unlikely that breastfeeding is the main underlying explanation. A recent study from Tanzania [19] reports that knowledge of optimal breastfeeding was higher among women receiving counselling on breastfeeding and infant feeding during antenatal care, compared to other women. However, these investigators did not detect an association between higher education and knowledge on optimal infant feeding [19]. In line with our findings, Patil et al reported that mother's education and age was not associated with duration of partial breastfeeding [2]. Thus, educational level, age and "experience" may play a more important role for child growth than the duration of breastfeeding, provided exclusive breastfeeding of at least 4 months and partial breastfeeding for a minimum of 12 months, which was reported in more than $3 / 4$ of children in our study

Our study had strengths and limitations. The main strength is the high number of participants, which was higher for this age group than the number of children from the same 
age group included from the Tanga region in the national nutrition survey of 2014 [15].

Moreover, none of the mothers declined participation and this reduces selection bias. We therefore believe our data are representative growth data for children between 12 to 24 months of age in the Tanga region in Tanzania. However, we cannot rule out that growth and health status of children not attending health clinics are different. A main limitation is a possible recall bias when it comes to data on breastfeeding. Mothers were interviewed about their breastfeeding practice and experience up to one year after they had stopped breastfeeding. They may not have detailed information on the exact duration of breastfeeding even though we had a clear impression that breastfeeding was considered important for these women. We investigated the change in WAZ over the last 5 months prior to study visit. Most infants attending the health clinics turned up each month for weighing. However, as we were lacking exact date of the prior visit we cannot rule out that for some children the first visit for comparisons may have occurred more than 5 months ago. As such, the percentage of children with decreasing WAZ may have been overestimated. Measuring height is also challenging using a measure tape, but all measurements were done by one person to increase consistency.

\section{CONCLUSION}

Mothers included in this cross-sectional study reported high rates of breastfeeding in first year of life and long duration of breastfeeding. However, children aged 12 to 24 months in the Tanga region of Tanzania had weight and height below WHO growth standards. Moreover, four out of 10 children in this age group had declining weight Z-scores over the last 5 months, often during a transition phase from exclusive and partial breastfeeding to solid foods.

Children of mothers who had higher educational showed better growth compared to those with low educational status. Further studies using both qualitative and quantitative approaches may identify more precisely reasons for nutritional problems and areas for improving nutrition in this area. 


\section{ACKNOWLEDGEMENT}

We would like to thank the health facility administrations for giving us permission to conduct this study in the two health clinics. We thank the interpreters for facilitating the interviews. We thank the nursing students from Tanga International Competence Centre for helping with anthropometric measurements. We thank Ruth Nesje for helping organise the stay in Tanga for the first author (ISE), and establishing contact between the study team and the health clinics. Most important, we thank all mothers and children for participating in this study.

\section{FUNDING}

There were no funding sources of this study.

\section{CONFLICTS OF INTEREST}

The authors declare no conflict of interest.

\section{ABBREVATIONS AND DEFINITIONS}

High maternal education

IQR

\section{LAZ}

Low maternal education

SD

WHO

WAZ

Z-score

$\Delta$-WAZ
- secondary school, college or university

- interquartile range

- length-for-age Z-score

- no education or only primary school

- standard deviation

- World Health Organization

- weight-for-age Z-score

- standard deviation score

- change in WAZ over the last 5 months 


\section{REFERENCES}

1. United Nations. The Millennium Development Goals Report 2015. New York.

http://www.un.org/millenniumgoals/2015_MDG_Report/pdf/MDG\%202015\%20rev\%20(July \%201).pdf

2. Patil CL, Turab A, Ambikapathi R, et al. Early interruption of exclusive breastfeeding: results from the eight-country MAL-ED study. J Health Popul Nutr. 2015;34:1-10.

3. Victora CG, Bahl R, Barros AJD, et al. Breastfeeding in the 21st century: epidemiology, mechanisms, and lifelong effect. Lancet. 2016;387:475-90.

4. Checkley W, Mouksassi S, Carreon DJ, et al. Childhood stunting in relation to the preand postnatal environment during the first 2 years of life: The MAL-ED longitudinal birth cohort study. PLoS Med. 2017;14:e1002408.

5. World Health Organization. Infant and young child feeding 2016.

http://www.who.int/mediacentre/factsheets/fs342/en/

6. Batiro B, Demissie T, Halala Y, et al. Determinants of stunting among children aged 6-59 months at Kindo Didaye woreda, Wolaita Zone, Southern Ethiopia: Unmatched case control study. PLoS One. 2017;12:e0189106.

7. Agostoni C, Braegger C, Decsi T, et al. Breast-feeding: A Commentary by the ESPGHAN Committee on Nutrition. J Pediatr Gastroenterol Nutr. 2009;49:112-25.

8. Lombardi J, Huang C, Madnick M, et al. 1.000 Days 2016. http://thousanddays.org/

9. Food and Agriculture Organization of the United Nations. FAO in emergencies. http://www.fao.org/emergencies/en/ (last accessed July 19, 2019)

10. United Nations Committee for Development Policy. List of Least Developed

Countries 2017. https://www.un.org/development/desa/dpad/wpcontent/uploads/sites/45/publication/ldc_list.pdf

11. World Health Organization. Child malnutrition country survey results 2015-2016. http://apps.who.int/gho/data/node.main.CHILDMALNUTRITION?lang=en

12. Nordang S, Shoo T, Holmboe-Ottesen G, et al. Women's work in farming, child feeding practices and nutritional among under-five children in rural Rukwa, Tanzania. Br J Nutr. 2015;114:1594-603

13. Sudfeld CR, McCoy DC, Fink G, et al. Malnutrition and Its Determinants AreAssociated with Suboptimal Cognitive, Communication, and Motor Development in Tanzanian Children. J Nutr. 2015;145:2705-14. 
s among under-five children in rural Rukwa, Tanzania. Br J Nutr. 2015;114:1594-603.

14. World Health Organization. The WHO Child Growth Standards 2006.

http://www.who.int/childgrowth/standards/en/

15. Assery O, Kaganda J, Kimboka S, et al. Tanzania National Nutrition Survey 2014. In:

Centre TFaN, editor. UNICEF: The United Republic of Tanzania, Ministry of Health and Social Welfare; 2014.

16. Semali IA, Tengia-Kessy A, Mmbaga EJ, et al. Prevalence and determinants of stunting in under-five children in central Tanzania: remaining threats to achieving Millennium Development Goal 4. BMC Public Health. 2015;15:1153.

17. Acosta AM, Chavez CB, Flores JT, et al. The MAL-ED Study: A Multinational and Multidisciplinary Approach to Understand the Relationship Between Enteric Pathogens, Malnutrition, Gut Physiology, Physical Growth, Cognitive Development, and Immune Responses in Infants and Children Up to 2 Years of Age in Resource-Poor Environments. Clin Infect Dis. 2014;59(suppl_4):S193-S206.

18. Spiegelman D, Liu E, Danaei G, et al. Active Tuberculosis in HIV-Exposed Tanzanian Children up to 2 years of Age: Early-Life Nutrition, Multivitamin Supplementation and Other Potential Risk Factors. J Trop Pediatr. 2015;62:29-37.

19. Hashim TH, Mgongo M, Katanga J, et al. Predictors of appropriate breastfeeding knowledge among pregnant women in Moshi Urban, Tanzania: a cross-sectional study. International Breastfeeding Journal. 2017;12:11. 
Figure 1. Change in weight-for age Z-score over the last 5 months ( $\Delta$-WAZ); decreasing (black), stable (white) or increasing (grey).

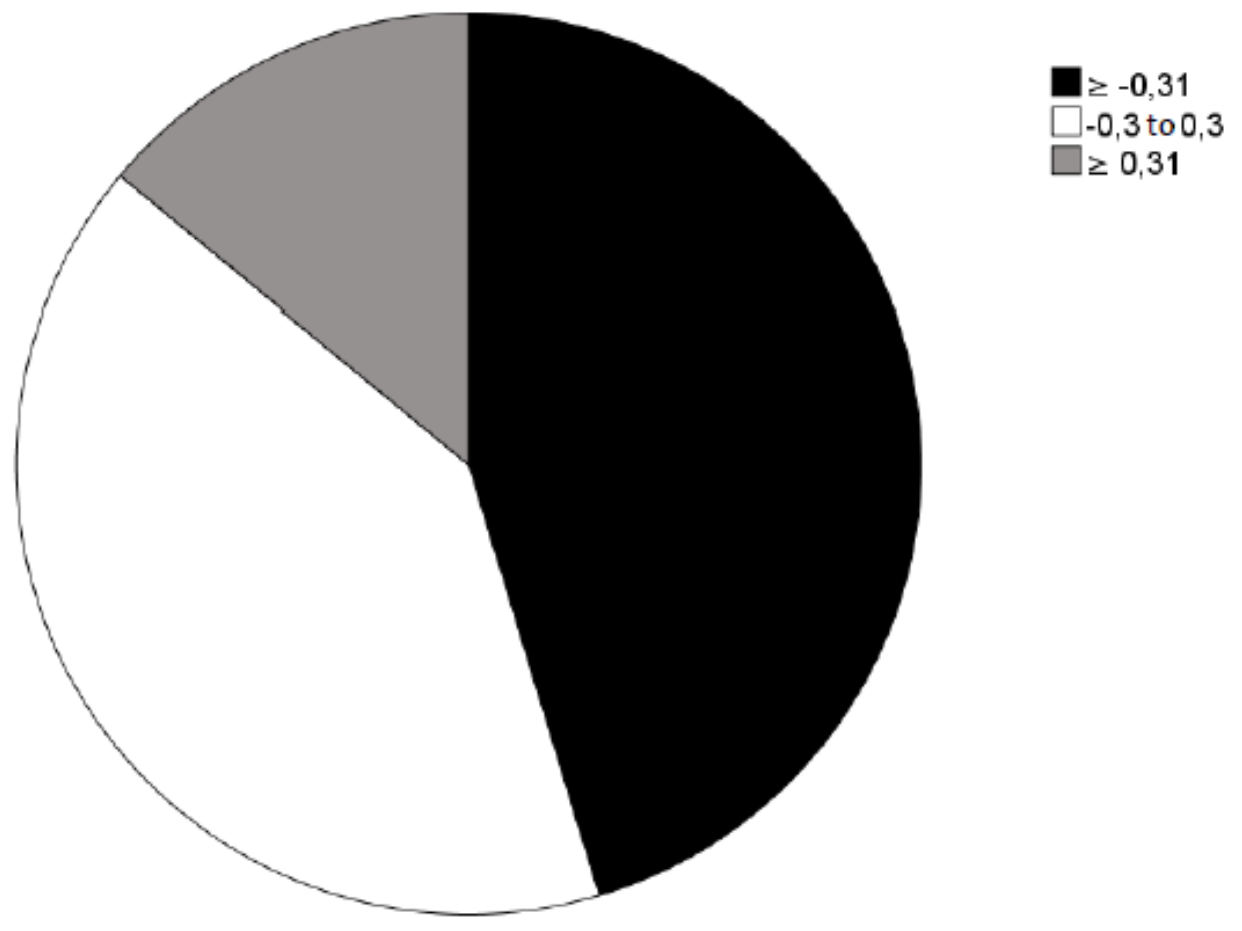


Table 1. Background data on 300 mother-child pairs included in this study.

\begin{tabular}{|c|c|}
\hline Mother & \\
\hline Age $(\text { years })^{a}$ & $27(23$ to 32$)$ \\
\hline Number previous pregnancies ${ }^{a}$ & $2(1-3)$ \\
\hline Number of births (incl. stillbirths) ${ }^{a}$ & $2(1-3)$ \\
\hline Number of children alive in the household ${ }^{\text {a }}$ & $2(1-3)$ \\
\hline \multicolumn{2}{|l|}{ Education } \\
\hline - None & $7(2.3 \%)$ \\
\hline - Primary & $180(60 \%)$ \\
\hline - Secondary & $104(34.7 \%)$ \\
\hline - University/college & $9(3.0 \%)$ \\
\hline \multicolumn{2}{|l|}{ Occupation } \\
\hline - Business & $141(47 \%)$ \\
\hline - Housewife & $139(46.3 \%)$ \\
\hline - Employee & $17(5.7 \%)$ \\
\hline - Peasant/student & $3(1 \%)$ \\
\hline Duration exclusive breastfeeding (months) ${ }^{a}$ & $6(4-6)$ \\
\hline - $0-3$ months & $70(23.3 \%)$ \\
\hline - 4-5 months & $54(18.0 \%)$ \\
\hline - 6 months & $170(56.7 \%)$ \\
\hline - $>6$ months & $6(2.0 \%)$ \\
\hline Reported breastfeeding $\geq 12$ months & $260(86.7 \%)$ \\
\hline Still breastfeeding on study visit $12-24$ months age & $217(72.3 \%)$ \\
\hline \multicolumn{2}{|l|}{ Child } \\
\hline Age at study visit (months) ${ }^{a}$ & $16(14-20)$ \\
\hline Female & $164(55 \%)$ \\
\hline Received all routine vaccinations & $290(97 \%)$ \\
\hline Weight-for-age Z-score (WAZ) ${ }^{b}$ & $-0.30(1.27)$ \\
\hline Length-for-age Z-score (LAZ) ${ }^{b}$ & $-0.47(1.67)$ \\
\hline Change in WAZ last 5 months ${ }^{b}$ & $-0.30(0.64)$ \\
\hline
\end{tabular}

${ }^{a}$ Median and interquartile range (IQR)

${ }^{\mathrm{b}}$ Mean and standard deviation (SD) 
Table 2. Maternal education and relation to breastfeeding and growth

\begin{tabular}{lccc}
\hline & \multicolumn{3}{c}{ Maternal education } \\
\hline & Low $(\mathrm{n}=187)$ & High $(\mathrm{n}=113)$ & $\mathrm{p}$ \\
\hline${\text { Duration exclusive breastfeeding }{ }^{\mathrm{a}}}$ & $6(3-6)$ & $6(4-6)$ & $0.85^{\mathrm{c}}$ \\
Weight-for-age Z-score $^{\mathrm{b}}$ & $-0.48(1.24)$ & $-0.00(1.28)$ & $0.001^{\mathrm{d}}$ \\
Length-for-age Z-score $^{\mathrm{b}}$ & $-0.66(1.7)$ & $-0.16(1.59)$ & $0.011^{\mathrm{d}}$ \\
Change in WAZ last 5 months $^{\mathrm{b}}$ & $-0.28(0.67)$ & $-0.32(0.60)$ & $0.63^{\mathrm{d}}$ \\
\hline
\end{tabular}

Low maternal education, none or primary school; high maternal education, secondary school or college/university

${ }^{\text {a }}$ Median and interquartile range (IQR)

${ }^{\mathrm{b}}$ Mean and standard deviation (SD)

${ }^{\mathrm{c}}$ Non-parametric Mann Whitney test

${ }^{\mathrm{d}}$ Student T test 
Table 3. Predictors possibly affecting attained growth in children age 12-24 months

\begin{tabular}{|c|c|c|c|c|}
\hline \multirow[b]{3}{*}{ Predictors } & \multicolumn{2}{|c|}{ Weight-for-age Z-score } & & \\
\hline & \multicolumn{2}{|c|}{ Univariate analysis } & \multicolumn{2}{|c|}{ Adjusted analysis $^{\mathrm{a}}$} \\
\hline & $\begin{array}{c}\text { Regression coefficient } \\
(95 \% \mathrm{CI})\end{array}$ & $\mathbf{p}$ & $\begin{array}{c}\text { Regression coefficient } \\
\text { (95\% CI) }\end{array}$ & $\mathbf{p}$ \\
\hline Maternal education & $0.48(0.19$ to 0.78$)$ & 0.001 & $0.57(0.27$ to 0.87$)$ & $<0.001$ \\
\hline Maternal age & $0.02(-0.001$ to 0.05$)$ & 0.06 & $0.03(0.01$ to 0.06$)$ & 0.008 \\
\hline Parity & $-0.09(-0.39$ to 0.21$)$ & 0.54 & & \\
\hline Duration exclusive breastfeeding & $-0.06(-0.14$ to 0.02$)$ & 0.16 & $-0.07(-0.15$ to 0.015$)$ & 0.11 \\
\hline Female & $-0.03(-0.33$ to 0.26$)$ & 0.82 & & \\
\hline Number of children in household & $-0.07(-0.19$ to 0.05$)$ & 0.26 & & \\
\hline Received all vaccinations & $0.58(-0.39$ to 1.54$)$ & 0.24 & & \\
\hline \multicolumn{5}{|c|}{ Length-for-age Z-score } \\
\hline & \multicolumn{2}{|c|}{ Univariate analysis } & \multicolumn{2}{|c|}{$\underline{\text { Adjusted analysis }}^{\text {a }}$} \\
\hline Predictors & $\begin{array}{c}\text { Regression coefficient } \\
(95 \% \mathrm{CI})\end{array}$ & $\mathbf{p}$ & $\begin{array}{l}\text { Regression coefficient } \\
(95 \% \mathrm{CI})\end{array}$ & $\mathbf{p}$ \\
\hline Maternal education & $0.50(0.11$ to 0.89$)$ & 0.006 & $0.50(0.11$ to 0.89$)$ & 0.012 \\
\hline Maternal age & $0.02(-0.01$ to 0.05$)$ & 0.29 & & \\
\hline Parity & $-0.13(-0.52$ to 0.26$)$ & 0.52 & & \\
\hline Duration exclusive breastfeeding & $-0.07(-0.18$ to 0.04$)$ & 0.19 & $-0.08(-0.18$ to 0.03$)$ & 0.17 \\
\hline Female & $0.14(-0.24$ to 0.53$)$ & 0.46 & & \\
\hline Number of children in household & $-0.09(-0.24$ to 0.07$)$ & 0.28 & & \\
\hline Received all vaccinations & $0.006(-1.25$ to 1.26$)$ & 0.99 & & \\
\hline
\end{tabular}

${ }^{a}$ Multiple linear regression, including predictors with $\mathrm{p}<0.20$ in the univariate model 
Table 4. Prevalence of stunted and underweight children identified in this study compared to data from the Tanzania National Nutrition Survey 2014 (15)*

Current study Tanga, National Nutrition

Tanzania 2017

Classification

Moderate underweight (WAZ $<-2$ SD and $\geq-3 \mathrm{SD})$

Severe underweight $(\mathrm{WAZ}<-3 \mathrm{SD})$

Moderate stunting (LAZ $<-2$ SD and $\geq-3$ SD)

Severe stunting $(\mathrm{LAZ}<-3 \mathrm{SD})$
(Age 12-24 months)

Survey, Tanzania 2014*

(Age 12-23 months)

$462 / 3640(12.2 \%)$

$135 / 3640(3.6 \%)$

$963 / 3626(25.2 \%)$

$16 / 300(5.3 \%)$ 